\title{
Comparison of Classical Nucleation Theory and Modern Theory of Phase Transition
}

\author{
Baranov SA ${ }^{1,2,3 *}$ \\ ${ }^{1}$ Institute of Applied Physics, Academy of Sciences of Moldova, Republic of Moldova \\ ${ }^{2}$ Department de Genie Physique, Ecole Polytechnique de Montreal, Montreal H3C 3A7, Quebec, Canada \\ ${ }^{3}$ Shevchenko Transnistria State University, Tiraspol, Republic of Moldova
}

\begin{abstract}
The formation of electrochemical phase is a typical example of a first-order phase transition. An overview on old and new thermodynamic and kinetic aspects of the theoretical description of first-order phase transitions, and, in particular, of the theory of nucleation is given. Electrochemical nucleation of nanostructures has been considered in terms of the classical Gibbs and the Cahn-Hilliard-Hillert theories. We obtained agreement between the theories Kahn-Hilliard-Hillert and Gibbs.
\end{abstract}

Keywords: Electrochemistry; Nucleation; Nanoparticles; Phase transitions; Gibbs theory; Cahn-Hilliard theory

\section{Introduction}

Electrochemical nucleation is usually described in the framework of the classical Gibbs theory of nucleation, which provides a simple expression for the critical radius of a growing nucleus (nanoparticles) [1-11]:

$$
r_{c}=K(\gamma / \mu)
$$

where $\gamma$ is the specific surface energy, $\mu$ is the change in volume energy during a phase transition (e.g., nucleation), and $\mathrm{K}$ is the particle shape factor.

The electrochemical kinetic reactions are determined by the magnitude of energy barrier $\Delta \mathrm{E}_{c}$ (activation energy) [5-9], which can be controlled by the surface energy, volume energy and shape factor. The steady flow rate of formation of the most probable nuclei can be defined as follows [3-9]:

$$
\mathrm{J} \sim \exp \left\{-\Delta \mathrm{E}_{\mathrm{c}} / \mathrm{k}_{\mathrm{B}} \mathrm{T}\right\}
$$

where $k_{B}$ is the Boltzmann constant, and $T$ is the absolute temperature.

The dimensionless parameter $\mathrm{G}$, which is also referred to as the Gibbs number, describes, equilibrium electrochemical nucleation and has the form [5]:

$$
\mathrm{G}=\Delta \mathrm{E}_{\mathrm{c}} / \mathrm{kT}
$$

Further, we briefly describe essential points of calculate $\Delta \mathrm{E}_{\mathrm{c}}$ adopted in the classical Gibbs model first and then address modern theory of phase transitions.

\section{Classical Gibbs Nucleation Theory}

Energy $\Delta \mathrm{E}_{\mathrm{c}}$ in the classical Gibbs nucleation theory (for a threedimensional case) was obtained by assuming that the nucleus is a sphere of radius $r$ [1-9]:

$$
\mathrm{G}_{3}=\mathrm{G}_{3 \mathrm{P}}-\mathrm{G}_{3 \mathrm{~V}}=4 \pi \mathrm{r}^{2} \gamma_{3}-(4 / 3) \pi \mathrm{r}^{3} \mu_{3}
$$

where $\gamma_{3}$ is the specific surface energy (for the three-dimensional case), $\mu_{3}$ is the change in volume energy during a phase transition (for the three-dimensional case).

The size of the critical radius of nucleation $r_{c}$ is determined in Equation 1 (where for the three-dimensional case: $\mathrm{K}_{3}=2$ ):

$$
r_{c}=2 \gamma / \mu
$$

Critical nucleus radius corresponding to maximum $G_{3}$ is as follows (Figure 1) [1-9]:

$$
\Delta \mathrm{G}_{3}=(16 \pi / 3)\left(\gamma_{3}^{3} / \mu_{3}^{2}\right)
$$

For a two-dimensional case, we obtain [1-9]

$$
\Delta \mathrm{G}_{2}=\mathrm{G}_{2 \mathrm{P}} / 2=\pi\left(\gamma_{2}^{2} / \mu_{2}\right)
$$

where $\gamma_{2}$ is the specific surface energy (for the two-dimensional case), $\mu_{3}$ is the change in volume energy during a phase transition (for the two-dimensional case).

The size of the critical radius of nucleation $r_{c}$ is determined in Equation 1 (where for the two-dimensional case: $\mathrm{K}_{2}=\mathbf{1}$ ):

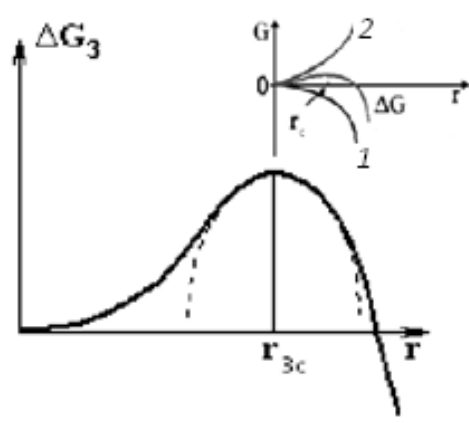

Figure 1: Qualitative kind diagram of the free energy barrier, $\Delta G_{3}$. The crossover of the bulk -1 and surface-2 terms combined with their opposing signs lead to the formation of a free energy barrier.

*Corresponding authors: Baranov SA, Department de Genie Physique Ecole Polytechnique de Montreal, Montreal H3C 3A7, Quebec, Canada, Tel: +37322731725; E-mail: baranov@phys.asm.md

Received August 23, 2017; Accepted August 28, 2017; Published August 30, 2017

Citation: Baranov SA (2017) Comparison of Classical Nucleation Theory and Modern Theory of Phase Transition. J Adv Chem Eng 7: 177. doi: 10.4172/20904568.1000177

Copyright: () 2017 Baranov SA. This is an open-access article distributed under the terms of the Creative Commons Attribution License, which permits unrestricted use, distribution, and reproduction in any medium, provided the original author and source are credited. 


$$
r_{2}=\gamma / \mu
$$
[1-9]

Critical nucleus radius corresponding to maximum $\mathrm{G}_{2}$ is as follows

$$
\Delta \mathrm{G}_{2}=\mathrm{G}_{2 \mathrm{P}} / 2=\pi\left(\gamma_{2}^{2} / \mu_{2}\right)
$$

The generalization can be obtained for a multidimensional system:

$$
r_{n c}=(n-1)\left(\gamma_{n} / \mu_{n}\right)
$$

And

$$
\Delta \mathrm{G}_{\mathrm{n}}=\mathrm{G}_{\mathrm{np}} / \mathrm{n}
$$

According to Equation 7, in one-dimensional case,

$$
\mathrm{r}_{1 \mathrm{c}}=0
$$

because $\mathrm{K}_{1}=0$.

This finding is consistent with the general theory. In terms of the droplet model, for convex surfaces, one can obtain the following expression:

$$
k(i) \sim \frac{S^{\prime}(r) r}{V^{\prime}(r)}
$$

To calculate energy barrier $\Delta G$, it is necessary to know the surface energy and particle shape factor $k(i)$ :

$$
\Delta \mathrm{G}_{\mathrm{i}} \sim \mathrm{G}_{\mathrm{P}} /[\mathrm{k}(\mathrm{i})+1]
$$

Energy barrier $\Delta \mathrm{G}$ can be assumed to be proportional to cathode overpotential $\eta_{\mathrm{k}}$ (see, e.g., $\left.[5,10,11]\right)$. This dependence can be presented as follows:

$$
\Delta \mathrm{G}_{n} \sim 1 /\left(\mu_{\mathrm{n}}\right)^{\mathrm{n}-1} \sim 1 /\left(\eta_{\mathrm{k}}\right)^{\mathrm{n}-1}
$$

It was experimentally studied for the case of $n=2$ and 3 , which follows from Equations 8-12 (see, e.g., [11]). Other general findings are given below. Here, we note that, if the short-range interaction covers the following focal areas, then radius $r_{n c}$ increases to infinity, while the energy barrier vanishes. It was found that, for spinodal disintegration, the radius (which is similar radius $r_{n c}$ ) increases indefinitely, while the energy barrier decreases.

\section{Ginsburg-Landau-Abrikosov Theory and Cahn- Hilliard Theory Applied to Nucleation}

The Ginsburg-Landau-Abrikosov theory uses free energy (Hamiltonian) in form [12-15]

\section{$\mathrm{H}(\Sigma)=\alpha(\nabla \Sigma)^{2}+\mathrm{A} \Sigma^{2}+\mathrm{C} \Sigma^{4}+13 \Delta$}

where order parameter $\Sigma$ is a generalized spinor (linear term is omitted).

Function $H(\Sigma)$ extreme condition allows deriving a differential equation. This equation was obtained in $[14,15]$ :

$$
\begin{aligned}
& \theta^{\prime \prime}(\rho)+((\mathrm{L}-1) / \rho) \theta^{\prime}(\rho)-\mathrm{F}[(\mathrm{a}, \rho), \cos \{\theta(\rho)\}, \sin \{\theta(\rho)\}]=014 \\
& 1 / \alpha=(\alpha / \mathrm{A})^{1 / 2}
\end{aligned}
$$

where $\mathrm{L}$ determines the space dimensionality of spinor $(\mathrm{nD}$ dimensionality), and $\mathrm{F}[(\mathrm{a}, \rho), \cos \{\theta(\rho)\}, \sin \{\theta(\rho)\}]$ is a polynomial (analytical function) that can be defined in each case.

Application of Equation 14 to a $2 \mathrm{D}$ problem was thoroughly considered in [15]. Below, we consider a $1 \mathrm{D}$ case $(\mathrm{L}=1)$, which corresponds to the Cahn-Hilliard-Hillert theory [16-22].
The resulting equation has the form

$\theta^{\prime \prime}(\rho)=(\mathrm{a} / 2) \sin \{2 \theta(\rho)\}(15)$

which is similar to previously reported equations [16-22] and according to which it is possible to obtain the solution [15]:

$$
\cos \{\theta(\rho)\}=-\operatorname{th}\{\mathrm{a} \rho)\}(16)
$$

The size of the domain wall is an important parameter determining the nucleating seed size (Figure 2):

$$
\delta_{\mathrm{LL}}=1 / \mathrm{a}=(\alpha / \mathrm{A})^{1 / 2}(17)
$$

Assuming that the domain wall size determines the critical radius of a nanoparticle, the difference between Equations 1 and 17 should be noted.

It can be shown that the energy of the domain wall is also different from the classical formulas given by Equations 5 and 8:

$$
\mathrm{E}_{\mathrm{LL}}=(\alpha \mathrm{A})^{1 / 2}(18)
$$

Agreement between the Ginsburg-Landau-Abrikosov (CahnHilliard-Hillert) theories and the classical Gibbs nucleation theory is discussed below.

\section{Unification of the Theories}

The simplest assumption regarding the relationship between the surface tension and the nanoparticle radius can be expressed as follows:

$$
\begin{aligned}
& \mathrm{r}_{\text {eff }} \approx \gamma / \mathrm{r}_{\text {rel }} \\
& \text { and }\left(\mathrm{r}_{\text {rel }}=\mathrm{r}_{\mathrm{c}} / \Delta_{\text {in }}\right)>1
\end{aligned}
$$

where $\Delta_{\text {in }}$ is the thickness of the surface layer of the nanoparticle and $\left(\mathrm{r}_{\mathrm{rel}}=\mathrm{r}_{\mathrm{c}} / \Delta_{\text {in }}\right)>1$ is a parameter of length characterizing the nanoparticle size in terms of $\Delta_{\text {in }}$.

If $r_{r e l}<1$, then the Gibbs theory is not applicable. However, it can be applied in accordance with the Cahn-Hilliard-Hillert theory. In this case, a formula similar to Equation 1 (or Equation 7 without taking into account the $\mathrm{k}_{\mathrm{n}}$ factor) can be written as follows:

$$
\mathrm{r}_{\mathrm{c}} \approx \gamma_{\mathrm{eff}} / \mu \approx \Delta_{\mathrm{n}} \gamma / \mathrm{r}_{\mathrm{c}} \mu
$$

It is not difficult to transit to the formulas in the form of Equation

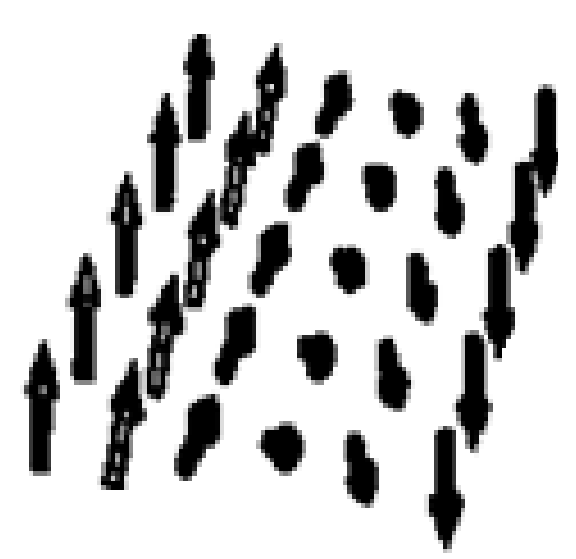

Figure 2: Schematic representation of the solution $[16,17]$ in the form of a domain wall of energy vectors (generalized spinor). 
Citation: Baranov SA (2017) Comparison of Classical Nucleation Theory and Modern Theory of Phase Transition. J Adv Chem Eng 7: 177. doi: 10.4172/2090-4568.1000177

Page 3 of 3

17 from the Cahn-Hilliard-Hillert theory:

$$
\begin{aligned}
& \mathrm{r}_{c}^{(\mathrm{KXX})} \approx\left(\Delta_{\text {in }} \gamma / \mu\right)^{1 / 2}, \\
& \text { If } \\
& \mathrm{K} \rightarrow \Delta_{\text {in }} \sigma_{\text {in }} \equiv \Delta_{\text {in }} \gamma ; \quad \mu \rightarrow \mathrm{G}_{\mathrm{KXX}}
\end{aligned}
$$

Thus, there is a correspondence between the Cahn-Hilliard-Hillert and Gibbs theories. It is of interest to show that there is no contradiction between the formulas for the activation energy in the Cahn-HilliardHillert and Gibbs theories.

In fact, the order of accuracy for activation energy is determined by the energy (barrier) necessary for merging particles, which is a product of the particle chemical potential $(-\mu)$ and the defect size (linear case implied) and can be estimated via Equation 21.

Finally, it is not difficult to arrive at the following formula:

$$
\Delta \mathrm{G} \sim\left(\Delta_{\text {in }} \gamma \mu\right)^{1 / 2}
$$

Therefore, there is no contradiction between the Cahn-HilliardHillert and Gibbs theories.

\section{Conclusions}

The formation of electrochemical phase is a typical example of a first-order phase transition. Electrodeposition of nanostructures has been considered in terms of the classical Gibbs and the Cahn-HilliardHillert theories. Agreement between the theories Kahn-HilliardHillert and Gibbs was obtained. We found no contradiction between the theories Cahn-Hilliard-Hillert and Gibbs. We have reviewed the progress of the modern thermodynamic theory of the growth of nanoparticles. We note the existence and of other approaches of the theory, presented for example in [23-26].

\section{References}

1. Gibbs JW (1928) Graphical methods in the thermodynamics of fluids. Yale University Press.

2. Frenkel J (1939) Statistical Theory of Condensation Phenomena. J Chem Phys 7: 200.

3. Frenkel J (1955) Kinetic Theory of Liquids. Dover Publications.

4. Zeldovich JB (1943) Acta Physicochim. U.R.S.S. 18: 1.

5. Gamburg Y, Zangari G (2011) Theory and Practice of Metal Electrodeposition. Springer, New York, USA, p: 375.
6. Volmer M, Weber A (1923) Z Phys Chem. (Leipzig) 119: 277.

7. Wagner CZ (1961) Theorie der Alterung von Niderschlagen durch Umlösen (Ostwald Reifung). Electrochem. 65: 581-591.

8. Anisimov MA (1991) Critical Phenomena in Liquids and Crystals. Philadelphia Gordon \& Breach, USA, p: 280.

9. Abraham FF (1974) Homogeneous Nucleation Theory. Academic Press, New York and London.

10. Erdey-Gruz T, Volmer MZ (1931) Phys Chem. 157: 165.

11. Vetter KJ (1967) Electrochemical Kinetics: Theoretical Aspects. Academic Press, New York, USA, p: 726.

12. Landau LD (1937) On the Theory of Phase Transitions 1. Phys Zs Sowjet 11 26.

13. Landau LD (1937) On the Theory of Phase Transitions 2. Phys Zs Sowjet 11: 545.

14. Ma SK (1976) Modern Theory of Critical Phenomena. Westview Press, USA.

15. Baranov SA (2015) Handbook of Nanoelectrochemistry: Electrochemical Synthesis Methods, Properties and Characterization Techniques. Springer, Switzerland, pp: 1057-1069.

16. Cahn JW, Hilliard JE (1958) Free Energy of a Nonuniform System. I. Interfacial Free Energy. J Chem Phys. 28: 258.

17. Cahn JW (1959) Free Energy of a Nonuniform System. II. Thermodynamic Basis. J Chem Phys 30: 1121.

18. Cahn JW, Hilliard JE (1959) Free Energy of a Nonuniform System. III. Nucleation in a Two $\square$ Component Incompressible Fluid. J Chem Phys 30: 688.

19. Cahn JW (1968) Spinodal decomposition. Trans Met Soc AIME 242: 166.

20. Cahn JW, Larche FC (1982) Surface stress and the chemical equilibrium of small crystals-II. Solid particles embedded in a solid matrix. Acta Met 30: 51.

21. Hillert M (1975) Diffusion and interface control of reactions in alloys. Metall Trans. 6: 5.

22. Khachaturyan AG (1983) Theory of Structural Transformations in Solids. Wiley, New York.

23. Schmelzer JWP (2002) In: Encyclopedia of Surface and Colloid Science. Marcel Dekker Inc., New York.

24. Gutzow I, Schmelzer J (1995) The Vitreous State: Thermodynamics, Structure Rheology, and Crystallization. Springer, Berlin, Germany.

25. Schmelzer JWP, Roepke G, Priezzhev VB (1999) Nucleation Theory and Applications. Joint Institute for Nuclear Research Publishing House, Dubna, Russia.

26. Schmelzer JWP, Roepke G, Priezzhev VB (2002) Nucleation Theory and Applications. Joint Institute for Nuclear Research Publishing House, Dubna, Russia. 